\title{
Comparing Ontology Authoring Workflows with Protégé: in the Laboratory, in the Tutorial and in the 'Wild'
}

DOI:

10.1016/j.websem.2018.09.004

\section{Document Version}

Accepted author manuscript

Link to publication record in Manchester Research Explorer

\section{Citation for published version (APA):}

Vigo, M., Matentzoglu, N., Jay, C., \& Stevens, R. (2018). Comparing Ontology Authoring Workflows with Protégé: in the Laboratory, in the Tutorial and in the 'Wild'. Journal of Web Semantics, 57, [100473].

https://doi.org/10.1016/j.websem.2018.09.004

\section{Published in:}

Journal of Web Semantics

\section{Citing this paper}

Please note that where the full-text provided on Manchester Research Explorer is the Author Accepted Manuscript or Proof version this may differ from the final Published version. If citing, it is advised that you check and use the publisher's definitive version.

\section{General rights}

Copyright and moral rights for the publications made accessible in the Research Explorer are retained by the authors and/or other copyright owners and it is a condition of accessing publications that users recognise and abide by the legal requirements associated with these rights.

\section{Takedown policy}

If you believe that this document breaches copyright please refer to the University of Manchester's Takedown Procedures [http://man.ac.uk/04Y6Bo] or contact uml.scholarlycommunications@manchester.ac.uk providing relevant details, so we can investigate your claim.

\section{OPEN ACCESS}




\title{
Comparing Ontology Authoring Workflows with Protégé: in the Laboratory, in the Tutorial and in the 'Wild'
}

\author{
Markel Vigo ${ }^{\mathrm{a}, *}$, Nicolas Matentzoglu ${ }^{\mathrm{b}}$, Caroline Jay $^{\mathrm{a}}$, Robert Stevens ${ }^{\mathrm{a}}$ \\ ${ }^{a}$ School of Computer Science, University of Manchester, Manchester, UK \\ ${ }^{b}$ European Bioinformatics Institute (EMBL-EBI), Hinxton, UK
}

\begin{abstract}
The development of ontology engineering tools has traditionally lacked a user-centred perspective, instead being guided by the need to address particular gaps indicated by anecdotal evidence. This has typically resulted in prototypes that do not obtain traction beyond a narrow scope. Understanding the authoring patterns of ontology engineers is crucial to informing the development of ontology engineering tools that cater for the activity workflows of the users and, consequently, boosting the adoption of these tools. We report evidence about how Protégé is used across three different authoring settings, addressing the threats to validity of relying on a single user study. These settings address the continuum of expertise (from intermediate to expert users), the type of tasks (whether they are free-form or prescriptive) and the effect of the location (laboratory, tutorial or on their own) and how the studies are administered (whether or not there is a close supervision). While there are activity workflows that are particular to settings, the results indicate a number of core workflows that are common to all of them. We discuss actionable recommendations for ontology engineering tools in light of these results.
\end{abstract}

Keywords: Empirical studies, Ontologies, usability, semantic web, authoring tools, engineering

\section{Introduction}

To date, ontology authoring tools have evolved in an ad hoc manner, supporting increasing levels of functionality, but not necessarily providing this support in an optimal way. While ontology engineering methodologies capture an anecdotal consensus about the types of activities that are commonly conducted by ontology engineers [26], deficiencies remain within existing tools that prevent ontology authors from being effective [29]. We claim that engineering tools have focused more on the functional requirements - and have been successful in doing so - at the cost of neglecting the user interface.

While there is early work that involved ontology authors in the design process of such engineering tools [4], it was not until recently that the user-centred design paradigm was systematically employed to better understand the human factors around ontology engineer-

\footnotetext{
${ }^{*}$ Corresponding author

Email addresses: markel.vigo@manchester.ac.uk (Markel Vigo), matentzn@ebi.ac.uk (Nicolas Matentzoglu), caroline . jay@manchester . ac . uk (Caroline Jay), robert.stevens@manchester.ac.uk (Robert Stevens)
}

ing and propose design recommendations for authoring tools through ethnographic observations [22], surveys [11, 37], interview studies [30] or controlled usability studies [31, 39].

We propose that an evidence-based approach to the development of ontology engineering tools should be taken. In a series of studies involving ontology authors [29, 31] we identified the existing gaps in current ontology authoring environments. One of these weaknesses, which was reported by ontology authors and empirically observed, was that users needed more effective tools to verify the consequences of their authoring activities. Individuals using the Inference Inspector [18], a Protégé plugin addressing these needs, were $42 \%$ more correct on their verification tasks than in the baseline system. This suggests that addressing authoring problems using evidence from empirical studies can potentially deliver a step change in the effectiveness of ontology engineering tools.

The goal of this paper is to give a comprehensive view of ontology authoring regularities across three different authoring settings (see Table 1) using Protégé, thus addressing the threats to validity of each of the settings. As there is no setting that maximises the internal and 
Table 1: Settings of the user studies

\begin{tabular}{lllll}
\hline Setting & Participants & Expertise & Tasks & Supervision \\
\hline Laboratory & 16 & Experts & Prescriptive & Close observation \\
Tutorial & 15 & Intermediate & Prescriptive-free-form & Semi-supervised \\
Remote naturalistic & 7 & Experts & Free-form & Non-supervised \\
\hline
\end{tabular}

external validity of the outcomes, we report user studies taking place (1) in a laboratory setting, (2) during a the tutorial about advanced features of OWL and (3) 'in the wild', i.e. while ontology authors build ontologies in their own environment. This way we address the weaknesses of one setting through the strengths of the others. By definition, controlled studies in the laboratory sacrifice external validity in order to maximise internal validity, so the remaining two studies address the following weaknesses of our original laboratory study [31]:

- Within a lab setting, response bias [21] and social desirability bias mean individuals try to meet the expectations of those who are administering the user study. While this bias is more prominent on self-reporting tasks, it can also potentially affect task performance.

- The Hawthorne effect [16], also known as the Guinea Pig effect [38], refers to individuals modifying their behaviour when they know they are being observed.

- The tasks set within a study constrain the external validity of the results. That is, results can be considered valid only under the goals, conditions and activities of these tasks. Whilst an effort is made to set 'typical' tasks, these are not exhaustive, or necessarily representative.

- Ontology engineering is a field for specialists: not only do the conventions of the languages have to be mastered (i.e. OWL, OBO, etc.), but also the tools of the trade and the application domain. Expertise is a spectrum, and in our first study we targeted individuals with high expertise, meaning the results may not be transferable to non-experts.

- The lack of familiarity with the ontologies used as stimuli might influence the performance of expert ontology authors, which is an undesirable outcome unless the goal is to carry out studies about learnability or knowledge acquisition. Since familiarity is acquired over time, multi-session studies could address this issue. One can work in domains with which most people are familiar (i.e. pizzas, or potato species as we did in our first study [31]). However, these toy domains may not adequately reflect all the complexities and nuances of ontology authoring.

By running more naturalistic and ecologically-valid settings we explore whether (1) the ontology authoring regularities we had discovered are exhibited universally; (2) there are other important patterns we had not yet found. In the lab study we were able to address the internal validity of the outcomes by controlling the confounding variables, and by triangulating our findings with our own observations, video recordings, interaction data and specialist machinery such as an eye-tracker. In this work we employ interaction logs generated by an instrumented version of Protégé, namely Protégé4US [30], in the naturalistic and tutorial studies, as it was not practical to use video-recording and eye-tracking technology.

This paper contributes to the advancement of ontology engineering as follows:

- We harvest interactions with Protégé in different settings to understand aspects of authoring behaviour in unexplored continua that may vary according to expertise, location, supervision and task.

- We find that, across settings, expert users are more efficient (the time elapsed between mouse clicks is shorter for them) and less hesitant (they exhibit fewer visuals search activities between clicks).

- We find evidence for a core set of workflow 1 that are common to the three settings we analyse including exploration of the asserted class hierarchy, reasoning, exploration of the inferred hierarchy and entity editing.

- We discover workflows that are particular to each setting, including the use of description logic

\footnotetext{
${ }^{1}$ When referring to 'workflows' we mean 'ontology authoring workflows', a subset of ontology engineering workflows comprising the activities performed to the actual building of the ontology.
} 
queries to check the latest modification (tutorial setting) and using the search functionalities of Protégé to find an entity that is going to be modified (naturalistic setting).

- We discuss the implications of our findings for the design of ontology authoring tools. For example, we propose that entity modification functionalities should be incorporated into search workflows. Also, our findings open up new avenues for user modeling and user interface adaptation in the context of ontology engineering.

\section{Related Work}

There are two main corpora of research that focus on how ontologies are built. There are those works that focus on the evolution of the contents of the ontology, and those that explore how tools interface with the authoring process. While our contribution is primarily to the latter, we acknowledge that it is difficult to disentangle these two aspects. The underlying infrastructure and tooling, and also the characteristic ways ontologies are represented through hierarchical arrangement of its classes and properties strongly determine how ontologies are explored and edited. Indeed, this is a common finding of previous works, where the class hierarchy not only gets $45 \%$ of the attention and activity of users [31], but also influences the editing behaviour of ontology authors [33].

Since the early days of knowledge engineering there have been calls for tools that would support users in carrying out typical tasks [19, 5]. Studies investigating this issue used questionnaires as the main mechanism for data collection [15, 6], which users filled out after completing a set of tasks defined by the researchers. The main finding of these early studies was that existing tools did not provide good enough support to users to accomplish the given tasks. A major criticism of these is their weak validity in that the small number of participants involved (fewer than eight users in some of the above works) and the typically lesser amount of information contained in data points collected through questionnaires does not guarantee enough statistical power to reliably support the claims made. The use of questionnaires for data collection can also result in unreliable data, due to the biases of self-reporting.

In order to address the concerns about the reliability we ran a controlled study in the lab where users conducted their tasks using Protégé4US [30]. Triangulating the $\log$ files generated by the interaction of users and eye-tracking data we (i) discovered workflows of exploration, edition and reasoning; (ii) found the class hierarchy is used as an index to explore the hierarchy; (iii) uncovered how predictive some sequences could be - e.g. running the reasoner after saving; and (iv) quantified how attention is allocated on the screen, where the class hierarchy gets $45 \%$ of the attention [31].

In a similar line of work that used interaction $\log$ files to understand the behaviours of ontology authors, Walk et al. [32] characterised people using their ontology exploration behaviours in BioPortal [20]. They identified seven types of users including main page visitors, who mainly browse the home page, search explorers, who extensively use the search functionality of the portal, and explorers of the ontology tree, whose navigation is guided by the hierarchical representation of the ontology. In another study the same authors found that the hierarchical arrangement of ontologies exercises a strong influence on ontology editing behaviour [33]. Interestingly, in another study on biomedical ontologies usage through the analysis of change logs, Wang et al. found that authoring behaviour is predictable (i.e. predicting what activity is going to happen next) as long as the ontology is known. This prediction is especially powerful when ontologies are at the maintenance stage, where the repertoire of exhibited behaviours is reduced [35]. This outcome confirms our finding of the predictability of some sequences of events and suggests that ontology authoring is an enterprise containing a significant number of regular activities. Predicting what is going to happen next is valuable, as it indicates that it is possible to provide assistance through incorporating recommender systems into ontology authoring workflows, and running time-demanding processes (e.g. running the reasoner) in the background before they are explicitly launched.

Often, ontology engineering is a collective and cooperative endeavour, so many studies have focused on the social dynamics of these collaborations. The work by Randall et al. [22] addresses the socio-technical issues of distributed collaborative authoring, highlighting that reaching consensus on the use, purpose and scope of a given ontology may generate tensions among the authors. While the findings emerged from an interview study, others have taken a quantitative approach by mining and clustering the interactions of a community of users [28]. The resulting clusters were considered behavioural profiles that grouped users by the frequency of their activity and the most frequent interactions. The modeled interactions were of a high-level granularity and could be understood as social processes whereby users made requests to the community such as "Request to add lexon" and "Constrain interaction request". An- 
Table 2: User interface events logged by Protégé4US

\begin{tabular}{ll}
\hline $\begin{array}{l}\text { Exploration events } \\
\text { Authoring events }\end{array}$ & $\begin{array}{l}\text { Hierarchy expanded, Hierarchy collapsed, Entity selected, Description selected } \\
\text { Class addition, Property addition, Individual addition, Annotation addition, Entity edited, Entity deleted, } \\
\text { Entity renamed, Set property, Entity dragged, Convert into defined, Convert into primitive } \\
\text { Environment events } \\
\text { Load ontology, Execute DL query, Run reasoner, Reasoner stop, Get explanation, Back, Undo, Redo, Save, } \\
\text { Click on usage, Search } \\
\text { Annotation hovered, Description hovered, Entity hovered, Explanation hovered }\end{array}$ \\
\hline
\end{tabular}

other work that analysed change-logs of collaborative engineering of large ontologies recommended that ontology engineering tools should implement role-based editing workflows [9]. This claim was supported by findings that indicated that different types of users exhibit different editing patterns: e.g. domain experts' contributions are focused on specific areas of ontologies, while the activities of ontology experts are not restricted to particular hierarchies, but are more evenly distributed.

As suggested by these works, we acknowledge that ontology authoring is often a collective and collaborative enterprise, where the exploration, search, edits and updates are conducted by individuals.

While early work focused on the appropriateness of tools to author ontologies by collecting data through observations and questionnaires, recent works have taken a more data-driven approach to study tool usage, ontology use and edition, and the socio-technical aspects of ontology engineering. However, while the mapping of the work done so far is promising - and independent works are confirming each other's findings - there are still unexplored questions regarding the internal and external validity of existing research.

While controlled laboratory experiments necessarily isolate variables to ensure the internal validity of the research, they are often criticised for their lack of ecological validity (see Section 1). To counteract this problem, we analyse the interaction logs generated by Protégé in settings that address some parts of the continuum of user expertise, tasks given, effects caused by the location of the ontology authoring activities and the role of those who administer the user studies. Our approach is ontology agnostic and draws on the tradition of early work, where the focus is more on the engineering environment, and less on the ontological artefact.

\section{Studies}

We ran three user studies to explore how the setting determines ontology authoring activities: Section 3.1 describes a controlled laboratory study, then in Section 3.2 we introduce a semi-controlled study run during an advanced OWL tutorial and, finally, in Section 3.3 we give details about a remote study. These three settings have characteristics that are placed at different ranges of the continua for participant expertise, task prescriptiveness and how the study is administered. Table 1 shows the methodological features of each study.

The participants of the three user studies employed Protégé4US [30] to conduct their ontology authoring activities. The events generated by Protégé4US are grouped into exploration events, authoring events, environment events and mouse hover events. Exploration events are those typically used to navigate the class and property hierarchy, and those related to the selection of entities in the hierarchy and their characteristics as shown in the 'Description' quadrant (see Figure 11. Note that exploration events can occur on asserted entities and on inferred ones. Authoring events are those that trigger the creation of new entities (classes, properties, individuals and annotations), their modification, deletion and rearrangement, and those actions that convert classes into defined classes through the equivalence axiom and primitive classes. Then, we have the events of the development environment and its plug-ins about loading ontologies, saving the current one, searching, executing queries, and events related to reasoning activity such as running the reasoner and showing the axioms that lead to a given inference through the 'Get explanation' command. Finally, we collect mouse hover events on asserted and inferred entities as indicators of activity. Table 2 shows the events that correspond to each group, most of which are self-explanatory.

\subsection{Laboratory setting}

The goal of this study was to author an ontology of potatoes to drive a 'potato finder' application. This domain was chosen so that previous domain knowledge would not influence the performance of participants. Indeed they reported a median of $1.5(\mathrm{SD}=1.7)$ when asked about it, where 1 indicated 'little knowledge about potato varieties' and 5 was for 'expert'. Participants were asked to carry out three tasks of increasing difficulty regarding the culinary role, yield and cropping time of potatoes. Participants were told not to start from scratch, but to adopt the persona of a 'jobbing' ontologist given an OWL ontology to extend and maintain. 


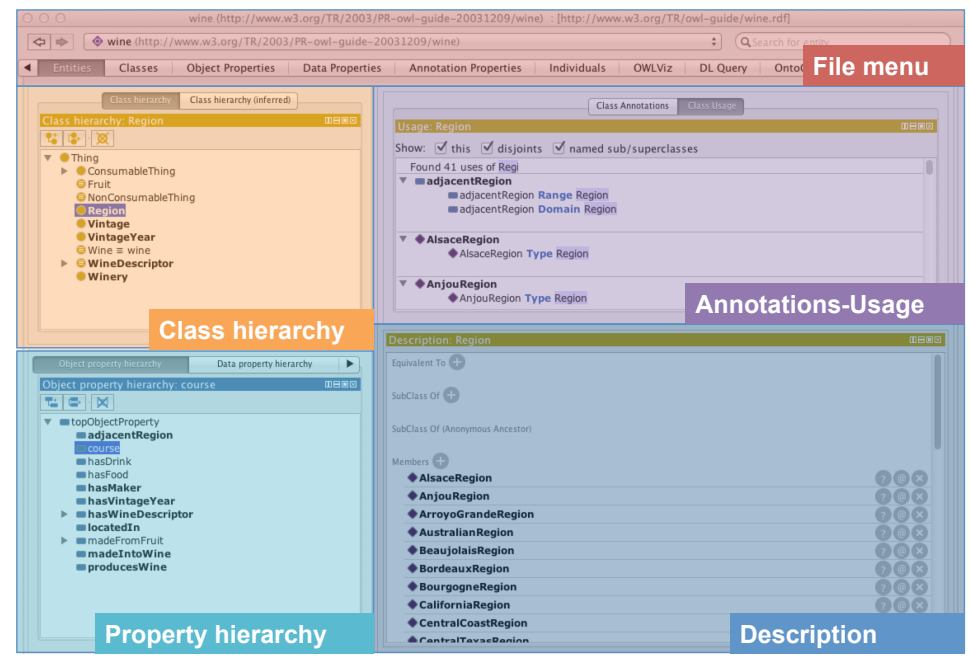

Figure 1: Core areas in Protégé

No explicit instructions were given on how to accomplish the tasks, but because participants were experts, we expected them to exhibit a repertoire of activities, including the invocation of the reasoner and the establishment of restrictions on classes. Participants were given a printed table with the necessary information including cropping time, yield, and skin colour to build the potato ontology. We also provided them with an OWL file containing 13 subclasses of various potato varieties and another one with a small hierarchy of cropping times for potatoes, which also removed some of the burden of large amounts of editing. There was no fixed time specified in which to complete the tasks, but participants were free to stop at any time. Full details of this study can be found elsewhere [31].

Participants. Sixteen individuals (eleven male) with a median age of 32.5 ( $\mathrm{SD}=7.2$ ) took part in the laboratory study. All of them had higher education degrees in Computer Science and three of them had a PhD. On a 5-point Likert scale assessing their expertise with OWL and Protégé a median score of 4 was reported for both questions ( $\mathrm{SD}=0.9$ and $\mathrm{SD}=0.6$ respectively), where 1 indicated 'novice' and 5 was for 'expert'.

\subsection{Tutorial setting}

The participants in this study took part in the Family History Advanced OWL Tutorial [24], which was taught by the authors of the paper. This tutorial targets individuals with a basic knowledge of the authoring environment (i.e. Protégé) and OWL that will enable them to build ontological artefacts of a certain sophistication. Hence, the completion of the more basic Pizza Tutorial was a precondition that was used as an inclusion criterion for those who wanted to participate.

The tutorial focuses on distinguishing between classes and individuals to later assert facts about individuals. It is extensive in the use of property hierarchies, property characteristics and role chains to drive inference, while running DL queries to check the outcomes of inference. While the tutorial was understandably prescriptive in the tasks participants had to accomplish in order to achieve the learning outcomes, there were many occasions in which participants had to experiment on their own to put into practice the theoretical aspects learned in the tutorial. Consequently we can say the tutorial setting may contain regularities that are brought about by prescriptiveness of the tasks, freeform activities they carried out on their own and other activities along the prescriptive-free-form continuum.

The tutorial was advertised on relevant mailing lists where it was indicated that the travel expenses were covered up to a certain amount. The tutorial was free and since all of them used Protégé4US, we gave them a $£ 10$ Amazon voucher if they gave us the consent to analyse the generated $\log$ files.

Participants. Fifteen individuals (six females) participated in the Advanced OWL Tutorial. Their median age was $34(\mathrm{SD}=13.3)$ and nine of them had a PhD. Eight of them had a biomedical background including zoology, biology, health and bioinformatics, six were computer scientists, two of them were mathematicians and one was an expert in geographical information systems - these categories are not exclusive. When asked about their current use of ontologies, eight participants 
responded that they dealt with lightweight ontologies and vocabularies, six said they worked with heavily axiomatised domain and application ontologies, and four responded that they used formal upper-level ontologies - again, these categories are non-exclusive. The domain of the ontologies participants had used was varied and ranged from neuroscience and biology to cartography, nutrition, transport and humanities. On a five point Likert-scale, where 1 indicated 'novice' and 5 was for 'expert' a median of 3 was obtained for expertise in OWL $(\mathrm{SD}=1.1)$ and Protégé $(\mathrm{SD}=1.2)$, which confirms that, as we mentioned earlier, these participants were not as expert as the ones in the lab study although they had been exposed to ontological work of a certain complexity.

\subsection{Remote naturalistic setting}

The participants of this study downloaded Protégé4US so that they could use Protégé on their PCs with their own ontologies and they were expected to work at their own pace. It is known that controlled remote studies (i.e. when tasks are administered by researchers at one end) are as good as conventional usability methods [1]. However, we deliberately decided not to intervene and not to give tasks (which we had done in the previous two studies) in order to observe authoring activities in as naturalistic a setting as possible.

We considered participants had finished the study when they had generated more than 15,000 events on the user interface of Protégé. Participants knew they had completed the study when the status bar of the study (implemented as a text label at the bottom-centre of the Protégé frame) changed from 'study on course' to 'study finished'. As soon as this flag changed they were free to stop from taking part and send us the log file generated on their PC. As the authoring pace of participants was variable some returned the log file in two days while some other did it after two weeks. Those who sent their $\log$ files were given a $£ 10$ Amazon voucher.

Participants. Seven individuals took part in this study (three females) with a median age of 33 (SD = 6.7). All of them had higher education degrees and four of them had a $\mathrm{PhD}$. They were recruited using snowball sampling whereby the inclusion criteria was that ontology authoring was central to the participants day-to-day work (hence, we did not ask them to report their expertise). This inclusion criteria distinguished these participants from those attending the Advanced OWL tutorial. Four of them considered themselves ontology researchers and the remaining ones indicated they were ontology developers. In terms of the type of work they conducted with ontologies, five worked with heavily axiomatised ontologies, five with lightweight ontologies and vocabularies and two of them dealt with formal upper-level ontologies (these categories were not exclusive). The domains people worked in were biomedical ontologies (five), chemistry and engineering (one) and media (one). Two of them used ontologies to run empirical experiments and reasoner based research.

\subsection{Analysis Methods}

In the three settings, in addition to the user interface events themselves, we also collected whether these occurred on asserted or inferred entities, and we distinguish between mouse hover events and events of interest (i.e. all the event that do not fall on the mouse hover category, henceforth eoi). We compute metrics as follows:

- To ascertain whether activity on inferred entities is different from that on asserted ones we compute the ratio of the number of activities on inferred entities to the number of activities on asserted ones: activity inferred $=\frac{n \text { (inferred events })}{n \text { (asserted events })}$. Values lower than 1 indicate more activity in asserted entities, while values greater than 1 indicate more activity in inferred entities. When comparing two values a higher value will be an indicator of more activity in inferred entities.

- Since mouse location on screen is a proxy of gaze location [12], it can be used to approximate visual exploration. We define it as the ratio of mouse hover events to the total number of events: exploration $=\frac{n(\text { mouse hover events })}{n \text { (mouse hover events })+n(\text { eoi })}$. Higher values of exploration indicate more visual search.

- We compute the mean elapsed time between two consecutive eoi in order to convey the tempo of ontology authoring, tempo $=\frac{1}{N-1} \sum_{i=2}^{N}\left(t_{i}-t_{i-1}\right)$, discarding periods of inactivity $>2$ minutes as they are likely to be due to interruptions ${ }^{2}$. Lower values of tempo indicate a more intense activity.

\footnotetext{
${ }^{2}$ Although longer periods of time are indicators of new sessions, we acknowledge this threshold may result in false negatives (i.e. if two consecutive eoi with a lag $>2$ minutes belong to the same session we would be splitting the session) although this would not affect the overall accuracy [14].
} 


\subsubsection{Identifying Authoring Patterns}

We apply N-gram pattern mining algorithms to the log files generated by Protégé4US in order to identify the most common sequences of events. The analysis is run on the events in the log files using the tau package [2] from the R statistical computing environment.

A common characteristic of the three settings and their corresponding datasets was the high number of contiguous repeated $\mathrm{N}$-grams of the same event, where an $\mathrm{N}$-gram of size 3 means there are 3 consecutive occurrences of the same event: e.g. class addition, class addition, class addition. Most of these N-grams of the same event are performed using a mouse click, which explains the abundance of the same consecutive events. However, this causes a noisy output and makes the identification of other meaningful sequences challenging [3]. To facilitate the analysis we merge the sequences of consecutive identical events, retaining a distinction between events consisting of a single occurrence and multiple consecutive occurrences of the same event. In the above example, this cleansing would result in the M_class_addition event when processing all the class addition events that occur consecutively, where the 'M' stands for 'multiple'. While this pre-processing of the log data facilitates our analysis, it should be noted that it assumes that all consecutive identical events - no matter if 2 or 17 - are semantically the same from an interaction modeling perspective.

On the pre-processed logs we computed $\mathrm{N}$-grams $\geq 3$ increasing the $\mathrm{N}$-gram size one at a time until the output of the computation only yielded concatenation of repeated and smaller $\mathrm{N}$-grams - notice that the greater the
$\mathrm{N}$-gram size one looks for, the fewer sequences are generated. Then, we selected the atomic $\mathrm{N}$-grams (i.e. small sequences that appear as part of longer $\mathrm{N}$-grams) as the representative ones and removed the permutations of the most frequent sequence when the permutations were not semantically different. For instance, we selected the sequence with the higher number of occurrences of the following two: <select entity, select description, modify entity $>$, <select description, modify entity, select entity>. In other cases such as <save ontology, run reasoner $>$ and $<$ run reasoner, save ontology $>$, these were considered two different sequences as different orders have different meanings. Finally, we identified the most frequent sequences.

\section{Results}

Figure 2 shows the distribution of events in each of the scenarios. While the magnitude of the frequencies is different, it can be observed that entity selection, entity edition, hierarchy expansion and description selection are always in the top-5 most frequent events. This reflects the fact that individuals use Protégé for exploring the ontology and modifying existing entities.

We observe that entity creation (i.e. class, property and individual addition) occurs more frequently in the remote study than in the tutorial or lab settings, where the modification of existing entities is more frequent than their creation. Interacting with annotations, and search tasks involving keyword typing and selection of results are two activities that happened in the remote setting that did not emerge in the laboratory or the tutorial.

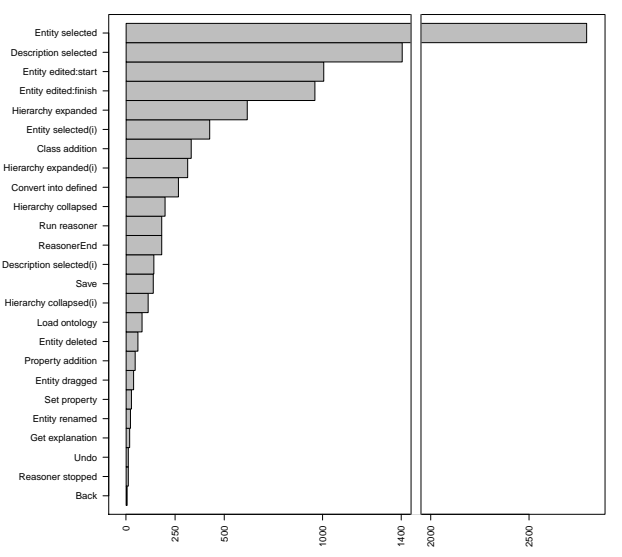

(a) Laboratory study

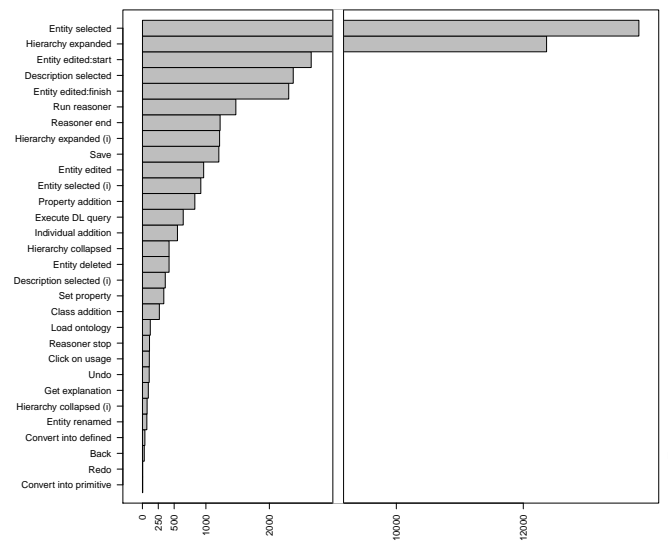

(b) Tutorial

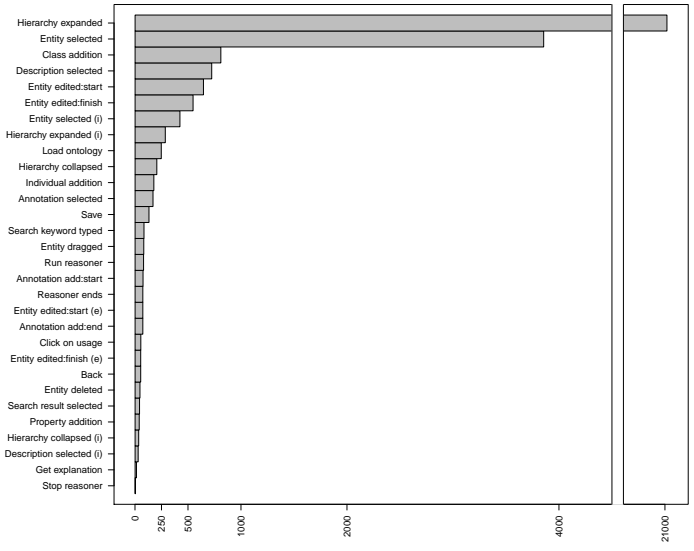

(c) Remote naturalistic

Figure 2: Frequency of user interface events on the laboratory $[a$, the tutorial $[b]$ and the remote study (c) 
Table 3: Descriptive statistics, tempo, exploration and activity inferred metrics for the three ontology authoring settings

\begin{tabular}{rccc}
\hline & $\begin{array}{l}\text { Laboratory } \\
(\mathbf{N}=16)\end{array}$ & $\begin{array}{l}\text { Tutorial } \\
(\mathbf{N}=15)\end{array}$ & $\begin{array}{l}\text { Remote } \\
(\mathbf{N}=7)\end{array}$ \\
\hline $\mathrm{N}_{\text {eoi }}$ & 9407 & 45121 & 30169 \\
Mean $_{\text {eoi }}$ & 588 & 3008 & 4309 \\
$\mathrm{SD}_{\text {eoi }}$ & 185 & 3030 & 7458 \\
\hline tempo & & & \\
Mean & $4.05-8.2$ & $7.26-12.35$ & $6.15-9.21$ \\
Median & $1.71-3.95$ & $2.78-4.63$ & $1.77-3.63$ \\
$\mathrm{SD}$ & $6.19-12.2$ & $12.81-19.87$ & $10.1-16.35$ \\
\hline exploration & & & \\
Mean & 0.85 & 0.89 & 0.77 \\
$\mathrm{SD}$ & 0.04 & 0.05 & 0.24 \\
\hline activity inferred & & & \\
Mean $_{\text {eoi }}$ & 0.12 & 0.13 & 0.12 \\
$\mathrm{SD}_{\text {eoi }}$ & 0.12 & 0.24 & 0.18 \\
Mean $_{\text {mhovers }}$ & $\mathrm{NA}$ & 0.18 & 0.16 \\
SD $_{\text {mhovers }}$ & $\mathrm{NA}$ & 0.43 & 0.20 \\
\hline
\end{tabular}

While it is understandable that there are no instances of interactions with annotations in the latter settings (due to the fact that they were not necessary for accomplishing the tasks), it is interesting that the search functionality was seldom used. This is probably due to the size of the ontologies, as it may have been straightforward to find existing entities in the small ontologies used in the lab and the tutorial, compared with the potentially larger ontologies used in the remote setting.

There are also events that are particular to the laboratory and tutorial settings. These are the use of the equivalence axiom through Convert into defined in the laboratory setting, and the execution of description logic queries and creation of instances of classes (i.e. individuals) in the tutorial. These two settings were also noted to be reasoning intensive, which accounts for $2 \%$ and $3.3 \%$ of the events of interest in the laboratory and tutorial respectively. Reasoning is not used as frequently in the wild but, again, is still relevant: it constitutes $0.2 \%$ of the events of interest and it is the 15th most frequent event.

Table 3 displays the total number of events of interest and the average number of times they occurred (and standard deviation) per participant as well as the mean, median (as data is skewed) and standard deviation for temp ${ }^{3}$ The results suggests that the authoring rhythm was quicker in the laboratory setting and slower in the

\footnotetext{
${ }^{3}$ We provide the range of these metrics as computing the mean of participants' means would have obscured the results.
}

tutorial. The pace on the remote setting is in between the other two settings with overlaps at both ends. This overlap is greater between the remote and lab setting and is confirmed by looking at the range of medians, which is very similar, although the variation within participants is larger for the remote setting.

When it comes to exploration, higher visual activity levels are observed in the tutorial and in the laboratory than in the remote setting although the latter shows a higher variability - note that higher values indicate more mouse activity. According to the values reported by $a c$ tivity inferred, the activity in inferred events of interest is very similar across settings (showing a higher variability in the tutorial), which indicates that $12-13 \%$ of the interactions occurred on inferred entities. This also applies to the mouse hover events over inferred entities, although in this case the percentage is situated around $16-18 \%$. In both cases the tutorial setting is $1-2$ percentage points above the other settings.

\subsection{Core Authoring Workflows}

The automaton on Figure 3 shows the most frequent $\mathrm{N}$-grams that emerged when we applied the method described in Section 3.4.1. The states convey user interface events in Protégé while the arrows between the states imply there is a probability $>0.2$ that two events occur consecutively - these probabilities are extracted from adjacency matrices that compute the number of transitions between any two states. The lack of an arrow between states does not indicate that no transitions occur, but rather that there is a lower probability of these. While our previous work focuses on the workflows and visual search strategies in the laboratory setting [31], the automaton in Figure 3 links together four atomic activities across the three settings. Hence, these four activities can be conceived as the lowest common denominator workflows.

Entity selection (typically the selection of an asserted class in the class hierarchy) is the state that acts as a hub between activities, meaning it is the event that is common to most activities. Most of the time participants reach this state by 1) loading an ontology into Protégé, and exploring the hierarchy by expanding and collapsing the class hierarchy (exploration activity - coloured in blue in Figure 3); 2) running the reasoner, which is often preceded by saving the current ontology (reasoning activity - orange); and 3) within cycles whereby entities are modified in a batch (editing activity - turquoise).

${ }^{4}$ NA values on Table 3 indicate that we did not collect this data in the laboratory setting as an earlier version of Protégé4US was used. 


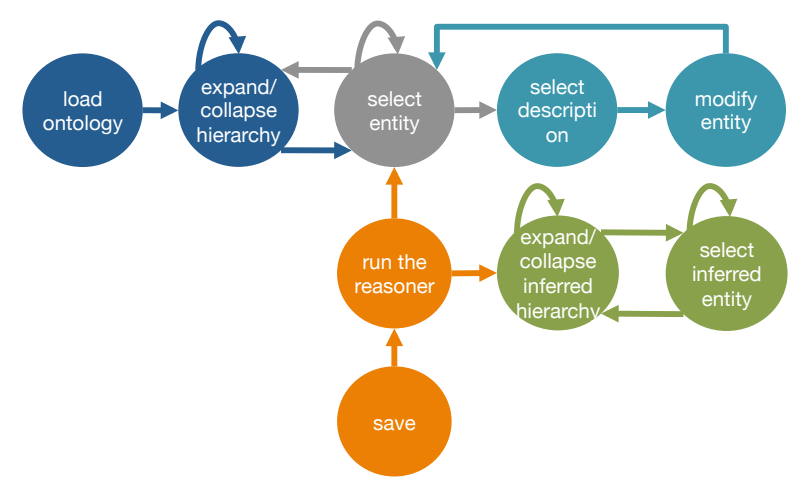

Figure 3: The core workflow: exploration of the asserted class hierarchy (blue), reasoning (orange), exploration of the inferred hierarchy (green) and entity editing workflow (turquoise)

With a probability of 0.3 both in the lab and the remote settings, users engage in the exploration of the inferred class hierarchy after running the reasoner while they interact with the inferred counterpart of the asserted entity selection event, examining inferred entities.

\subsubsection{Comparison of Workflow Completion Times}

The data driven analysis yielded the workflows that were common across the three settings. Once the workflows are known, this allows for computing specific features of the workflows including completion times, i.e. how long it takes to realise the activity. We define the workflows as the following sequences:

- Explore hierarchy: select entity $\leftrightarrow$ expand hierarchy, where either activity can be the start node.

- Explore inferred hierarchy: select inferred entity $\leftrightarrow$ expand inferred hierarchy, where either activity can be the start node.

- Load ontology and explore: load ontology $\rightarrow$ explore hierarchy.

- Reason and explore hierarchy: run the reasoner $\rightarrow$ explore hierarchy.
- Reason and explore the inferred hierarchy: run the reasoner $\rightarrow$ explore inferred hierarchy.

- Modify entity: select entity $\rightarrow$ select description $\rightarrow$ modify entity.

Note that workflows do not have a fixed number of activities as there are reflexive transitions in some nodes, and some edges are undirected as indicated by the automaton in Figure 3 Table 4 contains median completion times per workflow across settings. Figure 4 shows the distribution of the completion times as well as whether the differences in completion times are statistically significant when we apply the Kruskal-Wallis test.

Our analysis suggests that the class hierarchy is explored in sessions of 3.21-7.54 seconds 5 It takes longer if the class hierarchy is explored after an ontology is loaded in Protégé, taking around 9.50-12.38 seconds. In theory, users at the remote setting, who are not subject to time constraints, may exhibit longer sessions. Yet, our analysis suggests otherwise: even if completion times are different, these differences are not statistically significant for these two exploration workflows. The exploration of the inferred class hierarchy takes a similar amount of time, 2.11-6.46 seconds, although differences are significant between the lab and tutorial ( $p \leq 0.05)$, and the remote setting and the tutorial $(p \leq 0.05)$, where participants in the tutorial spent significantly longer time exploring the inferred class hierarchy.

Differences across settings become especially significant in the exploration workflows that are exhibited after running reasoner - see Figure $4 \mathrm{~d}$ and Figure $4 \mathrm{e}$ The tutorial shows higher completion times (26.27-38.82 seconds), compared to the lab (6.14-8.17 seconds) and the remote setting (11.43-19.85 seconds) with longer times on the inferred hierarchy, but in the tutorial setting. Fi-

${ }^{5}$ We report median times here. Standard deviations and Figure 4 display how data is spread.

Table 4: Median and standard deviation of core worflows per setting

\begin{tabular}{lrrrrrr}
\hline & \multicolumn{2}{c}{ Laboratory } & \multicolumn{2}{c}{ Tutorial } & \multicolumn{2}{c}{ Remote } \\
& Mdn & SD & Mdn & SD & Mdn & \multicolumn{1}{c}{ SD } \\
\hline Explore hierarchy & 7.54 & 14.81 & 3.67 & 264.59 & 3.21 & 153.62 \\
Explore inferred hierarchy & 2.11 & 7.90 & 6.46 & 121.17 & 2.48 & 21.43 \\
Load ontology and explore & 9.50 & 11.07 & 10.80 & 76.19 & 12.38 & 22.94 \\
Reason and explore hierarchy & 6.14 & 13.76 & 38.82 & 400.29 & 11.43 & 439.57 \\
Reason and explore the inferred hierarchy & 8.17 & 13.89 & 26.27 & 446.89 & 19.85 & 33.82 \\
Modify ontology & 7.91 & 35.01 & 9.28 & 196.22 & 10.83 & 208.69 \\
\hline
\end{tabular}


(a) Explore the hierarchy

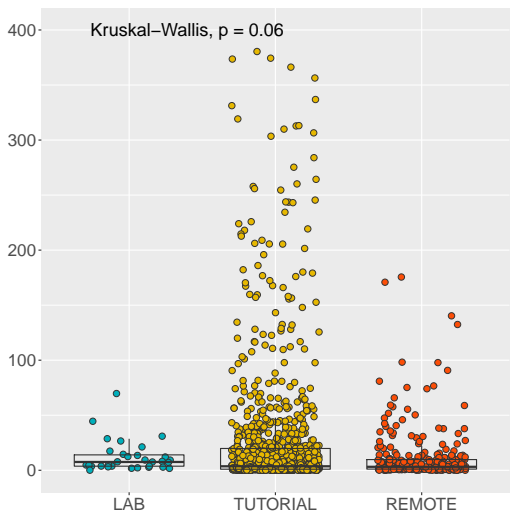

(d) Reason and explore the hierarchy

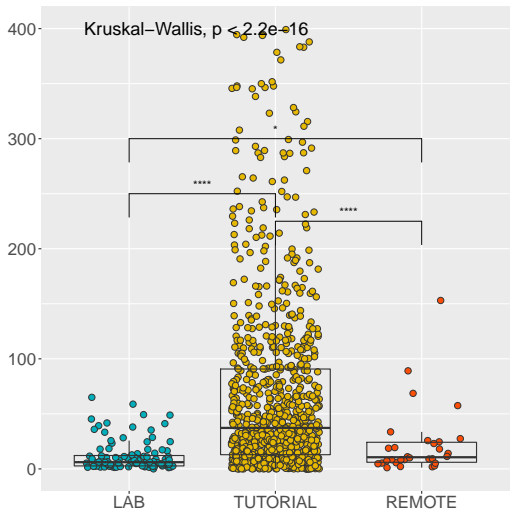

(b) Explore the inferred hierarchy

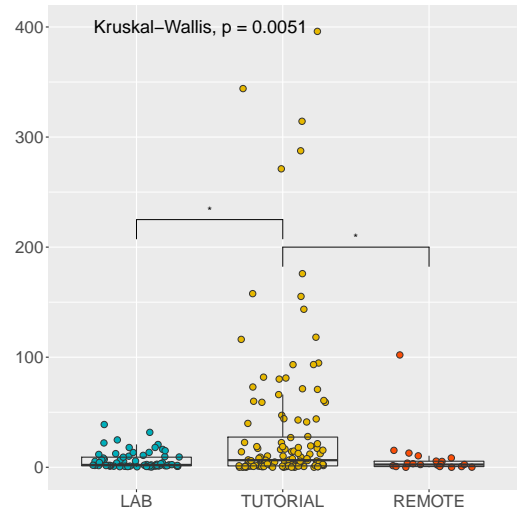

(e) Reason and explore the inferred hierarchy

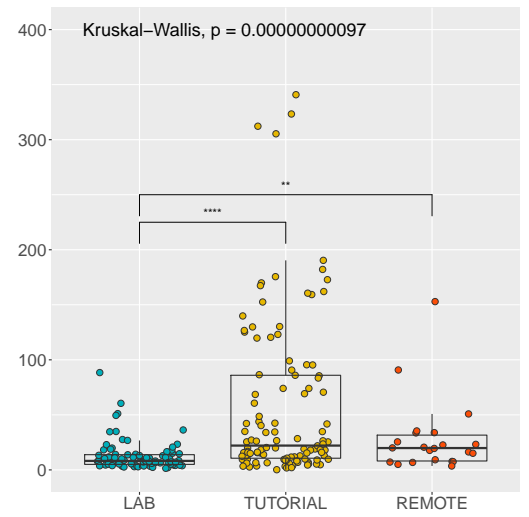

(c) Load ontology and explore the hierarchy

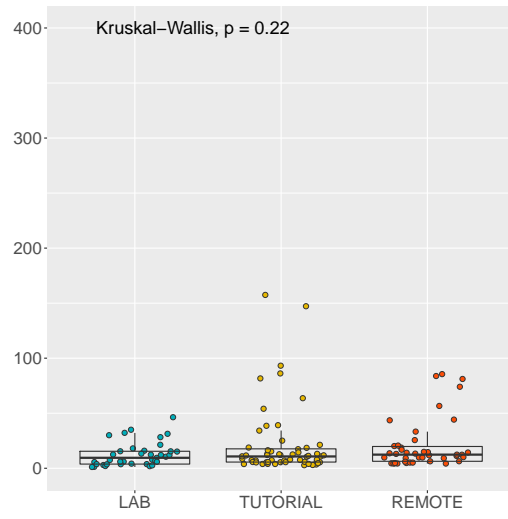

(f) Modify entity

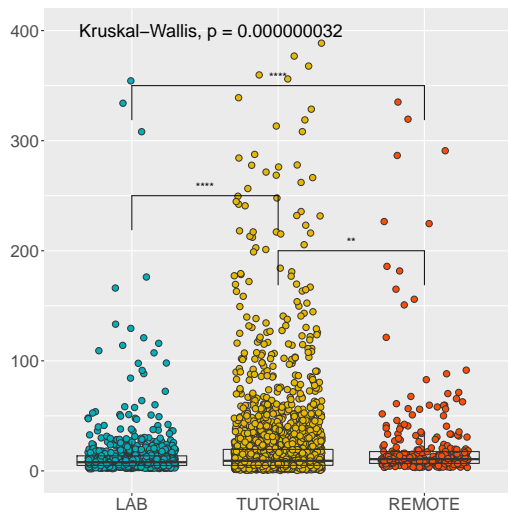

Figure 4: Distribution of workflow completion times per setting. Significance levels at $*: p \leq 0.05, * *: p \leq 0.01, * * *: p \leq 0.001, * * * *: p \leq 0.0001$

nally, participants show similar completion times (7.9110.83 seconds) in the entity modification workflow (see Figure 4), although differences are significant between the lab and tutorial $(p \leq 0.0001)$, lab and remote $(p \leq$ $0.0001)$, and tutorial and remote $(p \leq 0.01)$. It is worth highlighting higher standard deviations, and thus higher variability, in the tutorial and remote settings.

According to Table 4 there are differences between exploring the asserted and inferred class hierarchy. These differences are only significant for the tutorial setting $(t=-2.2, p<0.05)$ when we compare the Explore hierarchy and Explore inferred hierarchy workflows. No significant differences are found for other settings or other workflows.

\subsection{Workflows in the Tutorial}

While the save $\rightarrow$ run the reasoner workflow is a core workflow across all settings, it is particularly important in the tutorial setting, as evidenced by the high probability of transitioning between these two activities (i.e. $50 \%$ and 519 instances), presumably because it constitutes the hub workflow that connects the workflows that are particular to the tutorial (see Figure 5):

- Modify an entity, run the reasoner and check the outcomes. There are 273 instances of modify entity $\rightarrow$ save $\rightarrow$ run the reasoner and 47 of save $\rightarrow$ run the reasoner $\rightarrow$ run $D L$ query. The reflexive transition on run DL Query indicates there is query reformulation with a high probability $30 \%$ of the times). We found 21 instances of a four item $\mathrm{N}$ gram starting with entity modification and ending with running a DL query. One of the reasons why running the reasoner is central to the workflows discovered in the tutorial setting is because the outcomes of inference indicated whether the exercises 


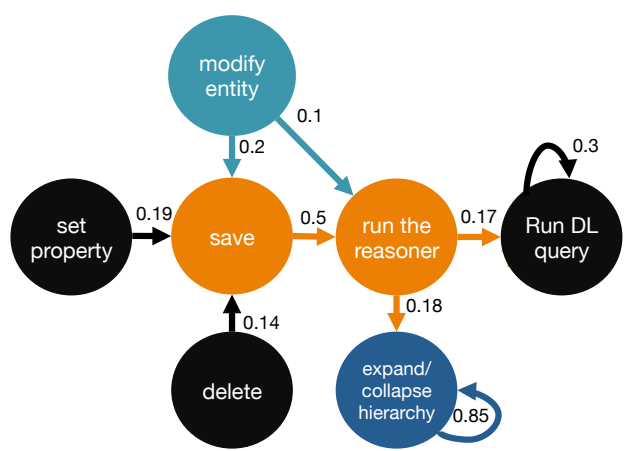

Figure 5: Authoring workflows exhibited in the tutorial study. Those states coloured in black are activities that do not belong to the core set and therefore are exclusive to the tutorial setting.

had been achieved successfully. In addition to navigating the inferred class hierarchy, which is a core workflow depicted in Figure 3, using DL queries helped participants to understand if they had met their goals.

- Delete entity, save and run the reasoner (55 instances). Due to the nature of the tutorial, we observed many instances where participants appeared to be trying to fix their mistakes. This 'trial and error' strategy worked by deleting the latest modification and running the reasoner to check whether the problem had disappeared. There are only two participants who used the back button (29 events), and while the use of undo is proportionately more frequent than in the remaining scenarios (107 times, $0.02 \%$ of all the events of interest) no regularities were found.

- Set a property to an entity, save and run the reasoner (32 instances). To an extent, this workflow reflects the tasks of the tutorial where an extensive use of properties and property chains was part of the teaching and the exercises.

- Save, run the reasoner and explore the class hierarchy (74 instances). This workflow suggests participants engaged with the exploration of the class hierarchy after running the reasoner. Since this is about navigating the asserted class hierarchy, this behaviour may have reflected an attempt to locate a particular class for later modification.

\subsection{Workflows in the Remote Setting}

The remote study confirms the findings of the laboratory and tutorial studies, and also extends them. In addition to the core workflows discussed earlier, four new

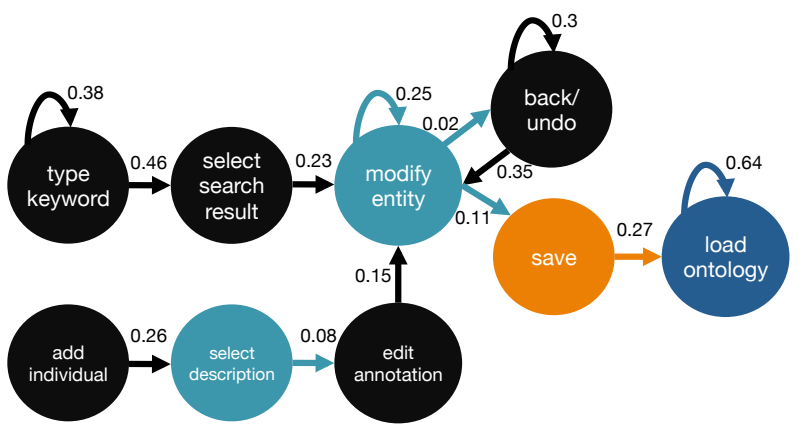

Figure 6: Authoring workflows exhibited in the remote study. Those states coloured in black are activities that do not belong to the core set and are therefore exclusive to the remote setting.

workflows are uncovered at the remote setting, which are shown in Figure 6. This time it is the modification of an entity that is the activity that acts as a hub and connects the following workflows:

- The search and modify workflow (92 instances). The search functionality is used to find entities that are going to be modified later. Search query refinements are observed, as $38 \%$ of the time after searching for a keyword another search is performed.

- Annotate individuals (75 instances). After adding individuals and selecting their description we find that participants add information about these individuals through annotations. Interestingly, while all the participants interacted with annotations, only two modified them.

- Heavy editing (120 instances), through modifying entities and undoing the latest modification. The number of transitions between entity modification and undo is 48 (the probability is $0.2 \%$ ) whereas there are 72 instances where participants modified an entity after undoing, meaning a probability of $35 \%$ of transitioning between these states. Notice the reflexive transition on undo, in that undo is followed by another undo command a third of the time.

- Moving onto the next task (42 instances). After modification of an entity, the ontology is saved and multiple ontologies are loaded in Protégé. We interpret this as the end of one task and start of the next one. Interestingly, we see multiple ontologies being loaded. 


\section{Discussion}

In the lab and tutorial settings, tasks were prescriptive about editing entities, and we thus believed that participants may have been doing more of this activity than they would in the wild. However, it was surprising to find that the remote setting was heavy on editing entities as well. As far as the creation of entities is concerned, one would expect that content creation would be less frequent than the modification of existing content due to the semantic web ethos on reusing existing entities. While this was true for the prescriptive settings ( 0.36 and 0.81 entities created per edit in the lab and the tutorial respectively), it was not for the remote setting, where we observe more entity creation (1028 entities: 810 classes, 40 properties and 178 individuals) than entity modification (548 edits), a ratio of 1.88 entities created per edit. While these ratios might be an artefact of the settings, they are also understandable in light of how entities are typically reused, which includes copying ontology statements from the original ontology (entailing the creation of an entity in the current ontology) and, optionally, establishing mappings with it [25]. The higher ecological validity of the remote setting yields activities not seen on the more controlled settings, including interactions with search and annotations.

A tempo of a higher frequency in the lab setting can be explained by the fact that the users could focus on accomplishing the tasks free from distractions. Whilst there was no time limit for doing the tasks, and the study was not conceived as a test, being observed may have added pressure to the situation, and influenced the authoring rhythm. This is in line with shorter workflow completion times in the lab setting. A surprising finding was that the tempo in the remote setting was of a higher frequency than in the tutorial. We could speculate that this could be due to the nature of the tutorial, but since we removed all periods of inactivity $>2$ minutes, this finding suggests that tempo is a proxy for expertise. That is, the quicker somebody clicks the mouse while conducting interactive activities the greater the confidence they have in their action. This phenomenon has been observed in Web navigation, where individuals with higher cognitive function can even click recklessly as they exhibit a repertoire of strategies to recover from unwanted outcomes [8].

Mouse exploration in Protégé is more intense in the controlled settings than in the remote one. This may indicate that the remote participants were less hesitant and more confident. It seems that, again, lower exploration levels might be associated with expertise. Since we defined exploration as a proxy for visual search - the location of the mouse is correlated with gaze location and therefore we can infer that the greater the number of mouse events the higher the gaze activity [12] we can say that those who are less expert exhibit more intensive visual search activities.

It was an unexpected finding that activities on inferred entities (conveyed by activity inferred) was almost the same across settings. We believed that the controlled settings were too prescriptive about the use of inference and the remote setting would have lower activity in this regard. Many ontologists (especially those dealing with large ontologies) either do not use the reasoner or use the reasoner very sparsely. Surprisingly we found a similar activity level in the remote setting, both for events of interest and mouse hover events. Yet, the prescriptive settings do make heavy use of the reasoner, accounting for $2-3 \%$ of the events (against the $0.2 \%$ on the remote setting). Significant differences were found between settings on workflows involving the exploration of the inferred class hierarchy, where participants exhibited higher completion times in the tutorial, especially when the exploration was realised after running the reasoner. Experienced users might know their ontologies well and are confident in what they are doing, so running the reasoner frequently to check the consequences of their edits may not be part of their repertoire of authoring strategies. It nevertheless appears that whilst reasoning is less frequent, the number of activities on the inferred entities is similar and more efficient.

Many more events were collected within the remote study. While this is desirable and was actively pursued by us, there is a downside to it: there is a higher variability as indicated by the $\mathrm{SD}_{e o i}$ values on Table 3 , which can make data harder to interpret.

\subsection{On the Workflows}

We find that the workflows exhibited in the lab also occur in the remote and tutorial settings: adding and editing restrictions to classes is the most common activity, exploration of the asserted class hierarchy is often a precursor to entity addition and modification, and the inferred hierarchy is explored after running the reasoner. These workflows emerged across settings where:

- The prescriptiveness of the tasks varied from totally prescriptive to free-form tasks.

- Participants reported levels of expertise along the intermediate-expert continuum.

- The administration of the study ranged from closely prescribed to completely unsupervised. 
Therefore we can say with a high level of certainty that the discovery of the core workflows across the three settings adds further evidence that supports their external validity. Common collaborative workflows were found across different large biomedical ontologies as well [34] when using WebProtégé [27]. Hence our finding reinforces the idea that there are some general principles that determine how ontologies are built, at least in Protégé. It is difficult to say whether these principles are influenced by engineering methodologies, or constrained by the functionalities and workflows brought about by tools. We hypothesise it is probably due to both although strongly determined by tools.

In addition to confirming a set of core Protégé workflows that were discovered in our laboratory study [31], we identify new behaviours we did not observe before. For instance, the use of search functionalities - sometimes involving refining the query - to locate an entity for modification was observed in the remote setting. This behaviour may be due to the fact that those in the remote setting were probably working with large ontologies including the Experimental Factor Ontology (EFO) [17], with 20460 classes, or the Chemical Entities of Biological Interest Ontology (ChEBI) [10] with 127337 classes. Using these ontologies as stimuli in the laboratory setting would have been unfeasible, as only participants with high domain expertise would have been able to accomplish the tasks.

We also found that the ontology is not only saved before running the reasoner, but also after heavy editing and before loading new ontologies into Protégé. This latter workflow may indicate the boundaries of different tasks or nervousness about the tool. The annotation of entities was observed among the participants of the remote setting. Adding annotations as soon as individuals are created was the most frequent workflow in our dataset. This suggests that human readable labels to entities has become good practice in the ontology's respective community, also because the development environment can render the label instead of the IRI if desired. Each setting has an activity that acts as a hub for the workflows. In the tutorial setting, most workflows include the save and run the reasoner activity, while in the remote setting entity modification is the hub activity. These hubs provide an indication of those activities that are representative of the settings.

Participants exhibited three main strategies for recovering from unexpected outcomes. In all the settings, updating the current ontology was realised through the constant modification of the ontology. In the tutorial, participants made heavy use of deletion to start from scratch again, and in the remote setting, undoing was the mechanism by which individuals refined their ontologies, as well as being a precursor of heavy editing. Interestingly, this strategy was not observed in the laboratory setting, even though expertise levels were similar. Undoing in the remote setting could have been exhibited due to the tasks being of a different nature, or the ontologies much greater in size - or perhaps to individual preferences.

\subsection{Implications}

The workflows we discovered tell us how individuals interact with Protégé and inform design recommendations for future versions as well as features that characterise ontology engineers.

\subsubsection{Implications for Design}

The workflows inform the following implications for design:

- Search as a first class citizen. Search for entities and individuals for their later modification is a two-stages workflow. This calls for seamlessly incorporating search functionalities within entity modification workflows. In order to link these two activities in a more effective manner, quick modifications could be enabled in search results.

- Prevent data loss. Due to the risk of reasoner crashes, it seems reasonable to protect the user against loss of data, either by asking to save before reasoning or saving the current ontology automatically so that it can then be restored when the application is restarted.

- Anticipate reasoner invocation. Users saving the current ontology before running the reasoner can be turned into an opportunity in that the reasoner could be run in the background to save time.

- Provide support for error recovery. We observed that users exhibited different strategies to adjust their authoring activities in order to achieve their goals: some by deleting parts of the ontology, others by using the undo functionality and most participants by updating the current ontology through the 'modify entity' workflow. Error recovery has two main components: finding the cause of the problem and deciding how to fix it. While these workflows for error recovery may be dependent on the expertise of the ontologists, their artefacts or just their preferences, they may signal authoring issues that may be better addressed by delivering 
interventions in real time through automated recommendations or online crowdsourced expert reviews.

- Keep a history of DL queries. DL queries are often used for evaluation purposes and users keep a set of them to check whether the latest modifications do not alter their expectations. This calls for keeping a history of DL queries that run in the current session so that users can run the ones executed earlier. Such a set of DL queries, associated with an ontology, could be viewed as a suite of tests for that ontology.

\subsubsection{Implications for User Modeling}

We found there are several proxies to characterise users with Protégé:

- Compared to expert users in the lab and remote settings, users with an intermediate expertise exhibited higher values of exploration, and thus of visual search. The amount of visual search is known to be an indicator of users encountering difficulties [7], which could well be an artefact of the tutorial, but also an indicator of their expertise.

- As suggested by the computed values of tempo, the pace in which the ontologies are built varies across settings and expertise levels. In the tutorial, tempo values were higher (indicating a slower pace) than in the lab and remote settings. While there might be an effect of the setting, higher activity rates (i.e. lower values of tempo) are indicators of more confidence and less costly strategies to recover from failure [8].

- Experts spend less time than intermediate users exploring the inferred hierarchy as indicated by the workflow completion times reported in Figure 4 One can argue that this is because the lab setting induce users to complete the tasks as soon as possible. However, no statistically significant differences are found between the lab and remote setting, where users are experts. This can be, again, an artefact of the tutorial setting although we do not discard the effect of user expertise.

These metrics could be used for keeping track of users' expertise in order to assess their progression and to adapt the user interface accordingly. Adaptations include (non-disruptive) switching between the axiom based view and the hierarchical relation-based view, and helping to address the need of role-based editing workflows [9].

\subsection{Methodological Considerations}

Internal validity. It can be questioned whether the number of participants (especially in the remote setting) constitutes a threat to internal validity. When it comes to usability evaluation, there is much discussion about the number of evaluators needed to make sure existing usability problems are detected [13, 23]. While some suggest that $10 \pm 2$ evaluators are enough [13], the number of evaluators depends on several variables including the purpose of the evaluation, the number of independent sessions per evaluator and the probability of not finding a usability problem [23]. First, our remote participants interacted with Protégé during periods ranging from two days to two weeks, exhibiting multiple sessions per user, which compensates for the number of participants. Second, the purpose of our work was to identify the regularities exhibited by ontology engineers and derive design improvements from these regularities. Therefore we do not need to be concerned with non-found usability problems but with non-encountered workflows. The workflows we found do exist and are valid, which is supported by the core workflows exhibited across all settings. It follows that the workflows we report might not be all the workflows that exist so we do not discard that further research may discover new ones.

External validity. While we acknowledge that there are ontologists that use other tools for ontology authoring purposes (or even ecosystems of different tools [29]), Protégé is still the authoring tool used by the vast majority of ontologists (around 74\% of ontology authors use it [36]). The implications for design are generalisable and transferable if other tools allow similar workflows to be exhibited. If the functionalities associated with workflows were absent, our implications for design would constitute research-driven opportunities to implement new functionalities - for a recent comparison of tools see [29].

The implications for user modeling are more generalisable in that the computed metrics (i.e. exploration and tempo) are, to a greater extent, independent of the workflows.

\section{Conclusion}

In a series of studies on ontology authoring, we identified the strategies employed by ontology authors to overcome the limitations of current ontology engineering tools [29] and isolated the authoring regularities exhibited in a controlled laboratory study with Protégé [31]. In this work, we find that there is a core 
workflow, which is common to different ontology authoring settings in continua that vary according to the expertise of users, their location, the amount of supervision and the prescriptiveness of the tasks. This does not only add further evidence that confirms our previous studies but provides external validity to previous and current findings.

Our outcomes inform design implications for ontology engineering tools in that the identified workflows and behavioural markers facilitate an evidence-based development of such tools. For instance, we find that the authoring rhythm, as well as the amount of visual exploration, are indicators of user expertise, which opens up new research avenues for user modeling, user interface adaptations and recommendations in the context of ontology engineering tools.

\section{Acknowledgments}

The user interaction logs analysed in this work are available at https://doi.org/10.5281/zenodo.1313020 This research has been funded by the EPSRC project: WhatIf: Answering "What if..." questions for Ontology Authoring. EPSRC reference EP/J014176/1.

\section{References}

[1] M. Andreasen, H. Nielsen, S. Schrøder, and J. Stage. What happened to remote usability testing?: An empirical study of three methods. In Proc. of the SIGCHI Conference on Human Factors in Computing Systems, CHI '07, pages 1405-1414, 2007.

[2] C. Buchta, K. Hornik, I. Feinerer, and D. Meyer. tau: Text analysis utilities. Available at http://cran.r-project.org/ web/packages/tau/

[3] H. Dev and Z. Liu. Identifying frequent user tasks from application logs. In Proceedings of the 22Nd International Conference on Intelligent User Interfaces, IUI '17, pages 263-273. ACM, 2017.

[4] A. Duineveld, R. Stoter, M. Weiden, B. Kenepa, and V. Benjamins. WonderTools? a comparative study of ontological engineering tools. International Journal of Human-Computer Studies, 52(6): 1111 - 1133, 2000.

[5] A. Duineveld, R. Stoter, M. Weiden, B. Kenepa, and V. Benjamins. WonderTools? a comparative study of ontological engineering tools. International Journal of Human-Computer Studies, 52(6): 1111 - 1133, 2000.

[6] M. Dzbor, E. Motta, C. Buil, J. M. Gomez, O. Goerlitz, and H. Lewen. Developing ontologies in OWL: An observational study. In OWL: Experiences and Directions Workshop, 2006.

[7] C. Ehmke and S. Wilson. Identifying web usability problems from eye-tracking data. In Proceedings of the 21st British $\mathrm{HCI}$ Group Annual Conference on People and Computers: HCI...But Not As We Know It - Volume 1, BCS-HCI '07, pages 119-128, Swinton, UK, UK, 2007. British Computer Society.

[8] P. G. Fairweather. How older and younger adults differ in their approach to problem solving on a complex website. In Proceedings of the 10th International ACM SIGACCESS Conference on Computers and Accessibility, Assets '08, pages 67-72, New York, NY, USA, 2008. ACM.
[9] S. Falconer, T. Tudorache, and N. F. Noy. An analysis of collaborative patterns in large-scale ontology development projects. In Proceedings of the Sixth International Conference on Knowledge Capture, K-CAP' 11, pages 25-32, New York, NY, USA, 2011. ACM.

[10] J. Hastings, P. de Matos, A. Dekker, M. Ennis, B. Harsha, N. Kale, V. Muthukrishnan, G. Owen, S. Turner, M. Williams, and C. Steinbeck. The ChEBI reference database and ontology for biologically relevant chemistry: enhancements for 2013. $\mathrm{Nu}$ cleic Acids Research, 41(D1):D456-D463, 2013.

[11] M. Horridge, T. Tudorache, J. Vendetti, C. I. Nyulas, M. A. Musen, and N. F. Noy. Simplified OWL Ontology Editing for the Web: Is WebProtégé Enough?, pages 200-215. Springer Berlin Heidelberg, Berlin, Heidelberg, 2013.

[12] J. Huang, R. White, and G. Buscher. User see, user point: Gaze and cursor alignment in web search. In Proceedings of the SIGCHI Conference on Human Factors in Computing Systems, CHI '12, pages 1341-1350, New York, NY, USA, 2012. ACM.

[13] W. Hwang and G. Salvendy. Number of people required for usability evaluation: The 10\&plusmn; 2 rule. Communications of the ACM, 53(5):130-133, May 2010.

[14] R. Jones and K. L. Klinkner. Beyond the session timeout: Automatic hierarchical segmentation of search topics in query logs. In Proceedings of the 17th ACM Conference on Information and Knowledge Management, CIKM '08, pages 699-708, New York, NY, USA, 2008. ACM.

[15] P. Lambrix, M. Habbouche, and M. Pérez. Evaluation of ontology development tools for bioinformatics. Bioinformatics, 19(12):1564-1571, 2003

[16] H. A. Landsberger. Hawthorne revisited: Management and the worker, its critics, and developments in human relations in industry. 1958.

[17] J. Malone, E. Holloway, T. Adamusiak, M. Kapushesky, J. Zheng, N. Kolesnikov, A. Zhukova, A. Brazma, and H. Parkinson. Modeling sample variables with an experimental factor ontology. Bioinformatics, 26(8):1112-1118, 2010.

[18] N. Matentzoglu, M. Vigo, C. Jay, and R. Stevens. Inference inspector: Improving the verification of ontology authoring actions. Web Semantics: Science, Services and Agents on the World Wide Web, 2017.

[19] D. L. McGuinness and P. F. Patel-Schneider. Usability issues in knowledge representation systems. In $A A A I / I A A I$, pages $608-$ $614,1998$.

[20] N. F. Noy, N. H. Shah, P. L. Whetzel, B. Dai, M. Dorf, N. Griffith, C. Jonquet, D. L. Rubin, M.-A. Storey, C. G. Chute, and M. A. Musen. Bioportal: ontologies and integrated data resources at the click of a mouse. Nucleic Acids Research, 37(suppl_2):W170-W173, 2009.

[21] D. L. Paulhus. Measurement and control of response bias. In Measures of Personality and Social Psychological Attitudes, chapter 2. Academic Press, 1991.

[22] D. Randall, R. Procter, Y. Lin, M. Poschen, W. Sharrock, and R. Stevens. Distributed ontology building as practical work. International Journal of Human-Computer Studies, 69(4):220 233, 2011.

[23] M. Schmettow. Sample size in usability studies. Communications of the ACM, 55(4):64-70, Apr. 2012.

[24] R. Stevens, M. Stevens, N. Matentzoglu, and S. Jupp. Manchester Family History Advanced OWL Tutorial. University of Manchester, 1.0 edition, 2013.

[25] M. C. Suárez-Figueroa. NeOn methodology for building ontology networks:specification, scheduling and reuse. Ontology Engineering Group, June 2010.

[26] M. C. Suárez-Figueroa, A. Gómez-Pérez, and M. Fernández- 
López. The NeOn methodology for ontology engineering. In M. C. Suárez-Figueroa, A. Gómez-Pérez, E. Motta, and A. Gangemi, editors, Ontology Engineering in a Networked World, pages 9-34. Springer Berlin Heidelberg, 2012.

[27] T. Tudorache, C. Nyulas, N. Noy, and M. Musen. Webprotg: A collaborative ontology editor and knowledge acquisition tool for the web. Semantic Web, 4(1):89-99, 2013. cited By 82.

[28] S. Van Laere, R. Buyl, M. Nyssen, and C. Debruyne. Detecting user profiles in collaborative ontology engineering using a user's interactions. Journal on Data Semantics, 6(2):71-82, Jun 2017.

[29] M. Vigo, S. Bail, C. Jay, and R. Stevens. Overcoming the pitfalls of ontology authoring: Strategies and implications for tool design. International Journal of Human-Computer Studies, 72(12):835-845, 2014.

[30] M. Vigo, C. Jay, and R. Stevens. Protégé4US: Harvesting ontology authoring data with protégé. In V. Presutti, E. Blomqvist, R. Troncy, H. Sack, I. Papadakis, and A. Tordai, editors, The Semantic Web: ESWC 2014 Satellite Events: ESWC 2014 Satellite Events, Anissaras, Crete, Greece, May 25-29, 2014, Revised Selected Papers, pages 86-99. Springer International Publishing, Cham, 2014.

[31] M. Vigo, C. Jay, and R. Stevens. Constructing Conceptual Knowledge Artefacts: Activity Patterns in the Ontology Authoring Process. In Proceedings of the 33rd Annual ACM Conference on Human Factors in Computing Systems, CHI 2015, Seoul, Republic of Korea, April 18-23, 2015, pages 3385-3394, 2015.

[32] S. Walk, L. Esín-Noboa, D. Helic, M. Strohmaier, and M. A. Musen. How users explore ontologies on the web: A study of
NCBO's bioportal usage logs. In Proceedings of the 26th International Conference on World Wide Web, WWW'17, pages 775-784, Republic and Canton of Geneva, Switzerland, 2017. International World Wide Web Conferences Steering Committee.

[33] S. Walk, P. Singer, L. E. Noboa, T. Tudorache, M. A. Musen, and M. Strohmaier. Understanding How Users Edit Ontologies: Comparing Hypotheses About Four Real-World Projects, pages 551-568. Springer International Publishing, Cham, 2015.

[34] S. Walk, P. Singer, M. Strohmaier, T. Tudorache, M. A. Musen, and N. F. Noy. Discovering beaten paths in collaborative ontology-engineering projects using Markov chains. Journal of Biomedical Informatics, 51:254 - 271, 2014.

[35] H. Wang, T. Tudorache, D. Dou, N. F. Noy, and M. A. Musen. Analysis and prediction of user editing patterns in ontology development projects. Journal on Data Semantics, 4(2):117-132, Jun 2015.

[36] P. Warren. Ontology users' survey - summary of results. Technical Report kmi-13-01, Knowledge Media Institute, 2013.

[37] P. Warren, P. Mulholland, T. Collins, and E. Motta. Using Ontologies, pages 579-590. Springer International Publishing, 2014.

[38] E. Webb, D. Campbell, R. Schwartz, and L. Sechrest. Unobtrusive Measures. Sage Publications, second edition, 2000.

[39] Y. Zhang, T. Tudorache, M. Horridge, and M. A. Musen. Helping users bootstrap ontologies: An empirical investigation. In Proceedings of the 33rd Annual ACM Conference on Human Factors in Computing Systems, CHI '15, pages 3395-3398, New York, NY, USA, 2015. ACM. 